\section{An unsual finding of brain magnetic resonance imaging in a hypertensive patient}

\author{
Harris A. Ngow, ${ }^{1}$ \\ Wan Mohd Nowalid Wan Khairina, ${ }^{2}$ \\ Bin Basri Hamidon ${ }^{3}$ \\ 'International Islamic University of \\ Malaysia, Kulliyah of Medicine, Kuantan, \\ Pahang, Malaysia; \\ ${ }^{2}$ Ministry of Health, Malaysia; \\ ${ }^{3}$ Hospital Universiti Kebangsaan Malaysia, \\ University Kebangsaan Malaysia, Jalan \\ Yaacob Latiff, Bandar Tun Razak, Cheras, \\ Kuala Lumpur, Malaysia
}

\begin{abstract}
Brain edema in patients with hypertensive encephalopathy frequently affects the parietooccipital white matter. Hypertensive encephalopathy is thus included as a differential diagnosis in reversible posterior leukoencephalopathy syndrome. Diffuse white matter involvement rarely occurs. We report a 41-year old woman with hypertensive encephalopathy with diffuse and non-enhancing white matter hyper-intensities throughout the whole brain on magnetic resonance imaging (MRI). These hyperintensities spared the grey matter on T2weighted and FLAIR sequence. These unusual finding on brain MRI was attributed to severe vasogenic cerebral edema resulting from accelerated hypertension.
\end{abstract}

\section{Introduction}

Hypertensive encephalopathy is an acute reversible neurological syndrome characterized by sudden onset, accelerated severe arterial hypertension, headache, confusion, convulsions and symptoms of compromise to posterior circulation. ${ }^{1-3}$ Brain edema occurs mainly in the white matter of the posterior cerebral region hence the term reversible posterior leukoencephalopathy syndrome (RPLS). Although this condition usually has a predilection for posterior white matter of the brain, the cerebral cortex, the frontal area, the brainstem, cerebellum and basal ganglia may also be involved. Extension of the edema into the brainstem, basal ganglia and cerebellum is almost always associated with cortical lesion. Diffuse white matter involvement is extremely rare. ${ }^{4}$ MRI more accurately visualizes these lesions compared to brain CT. ${ }^{5,6}$ This syndrome is often associated with abrupt increase in blood pressure usually seen in patients with malignant hypertension, eclampsia and renal disease. It is also seen in patients treated with cytotoxic and immunosuppressive agents such as cyclosporine, tacrolimus, and interferon $\alpha$. We describe an unusual case of hypertensive encephalopathy with brain MRI showing extensive deep white matter leukoencephalopathy involving the whole brain.

\section{Case Report}

A 41-year-old Malay woman with no known illness presented with an acute right-sided flank pain associated with nausea and vomiting of 1-day duration. She denied lower urinary tract symptoms, headache, chest pain, rashes, joint pain, seizures or dyspnea. She was at first day of menstruation at the time of presentation. She had history of pregnancyinduced hypertension during her first pregnancy and her blood pressure normalized after delivery. She had five children and her last childbirth was six years ago. There was no family history of hypertension or sudden cardiac death. She was not a smoker and did not consume alcohol, use recreational drugs or traditional medicine.

On examination, she was fully conscious but restless and irritable. She was pale but not jaundiced. Her blood pressure on admission was $275 / 151 \mathrm{mmHg}$. She was afebrile. Cardiovascular examination revealed signs of mild heart failure with cardiomegaly. She had papilloedema with flame shaped hemorrhages. Central nervous system examination and other systemic examinations were unremarkable.

Her laboratory test showed acute renal failure with serum creatinine of $607 \mu \mathrm{mol} / \mathrm{L}$ and urea of $23.6 \mathrm{mmol} / \mathrm{L}$. Her initial urinalysis showed mild proteinuria, bacteriuria, and pyuria with abundant red blood cells. She was empirically treated with broad-spectrum antibiotic for her urinary tract infection. Cardiac troponin T was elevated and electrocardiogram showed evidence of non-ST elevation myocardial infarction. Echocardiography showed good left ventricular function $(\mathrm{EF}=60 \%)$ with marked left ventricular hypertrophy. A brain computed tomography revealed a diffuse cerebral, cerebellar and brainstem white matter hypo-density (Figure 1). Brain MRI on day 2 of admission revealed diffuse and extensive non-enhancing hyper-intense lesion involving the whole brain including cerebral white matter, cerebellar white matter, thalami, basal ganglia, brainstem and middle cerebella peduncle on T2 weighted and FLAIR sequence with sparing of the grey matter (Figure 2). She was treated with intravenous nitroglycerine and subsequently required multiple oral antihypertensives to control her blood pressure. She was finally discharged from the hospital
Correspondence: Harris A. Ngow,

International Islamic University Malaysia, Kulliyah Of Medicine, Kuantan, Pahang, Malaysia E mail: harrisngow@gmail.com

Key words: cerebral edema, vasogenic, hypertensive encephalopathy, magnetic resonance imaging, reversible posterior leukoencephalopathy syndrome.

Received for publication: 13 March 2009 Accepted for publication: 25 April 2009.

This work is licensed under a Creative Commons Attribution 3.0 License (by-nc 3.0)

@C Copyright Harris A. Ngow et al., 2009

Licensee PAGEPress, Italy

Neurology International 2009; 1:e3 doi:10.4081/ni.2009.e3

with a well-controlled blood pressure after nine days. Connective tissue disease screening was normal. Further investigations for secondary hypertension were normal. The hypertension was accelerated by urinary tract infection which precipitated the acute renal failure. Her renal function, however, improved remarkably. One month later, a repeat ophthalmology review showed improvement of the papilloedema. Ten months after presentation, the patient continued to be well with normal neurological assessment. A repeat brain MRI showed partial resolution of the white matter lesions with some areas of infarct (Figure 3).

\section{Discussion}

Although the exact etiopathogenesis of hypertensive encephalopathy is not known precisely, the pathophysiological changes are associated with reversible vasogenic edema This may result from a rapidly rising blood pressure to overcome the brain's normal autoregulation of cerebral blood flow. This disturbance of autoregulation produces dilatation of cerebral arterioles with opening up of endothelial tight junction and leakage of plasma and red cells into extracellular space producing cerebral edema. ${ }^{7,8}$ The cerebral white matter is composed of myelinated fibres in a cellular matrix of glial cells, arterioles and capillaries that make this structure more susceptible to accumulation of fluid in the extracellular spaces leading to vasogenic edema. ${ }^{5}$ The adrenergic sympathetic innervation of the cerebral blood vessels is also an important component in the physiological mechanism of cerebral blood flow autoregulation. The carotid circulation is better supplied with adrenergic 

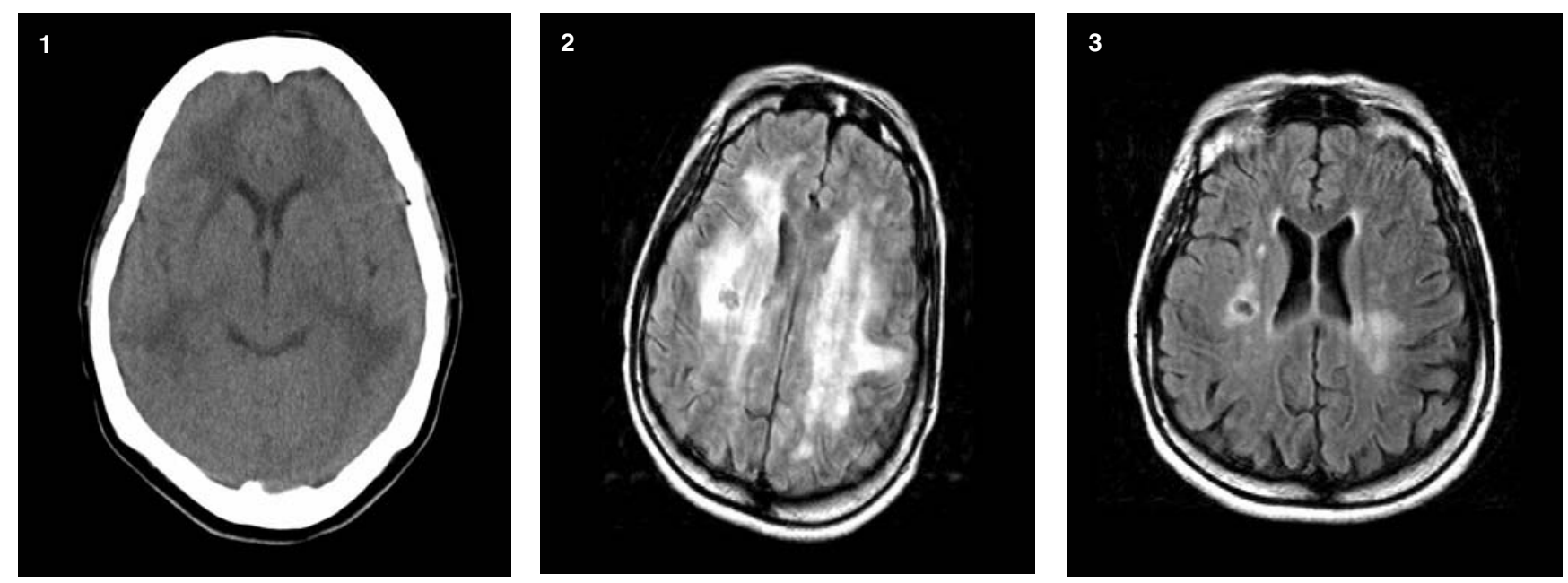

Figures 1-3. Figure 1 and 2 show the extensive leukoencephalopathy. Figure 1 shows the cranial computed tomography with bilateral and symmetrical hypodensities involving white and grey matter. Figure 2 is the FLAIR image that showed the extensive diffuse white matter signal abnormalities throughout the brain. Figure 3 shows the FLAIR sequence of cranial magnetic resonance imaging with resolution of the extensive white matter abnormalities.

innervations than the vertebro-basilar system. This inherent deficiency of adrenergic innervation may result in loss of vasoconstrictor properties of cerebral blood vessels mainly in the posterior cerebral area. ${ }^{9}$ However, the distribution of the edema seems to correlate well with both the severity and duration of blood pressure elevation. ${ }^{5,6}$ In mild acceleration of hypertension, edema is produced in the supratentorial white matter with little or no involvement of infratentorial compartments. Severe acceleration of hypertension produces more extensive supratentorial edema with extension into the brainstem, basal ganglia and cerebellum. The mean arterial pressure of this patient exceeded $190 \mathrm{mmHg}$, which suggests that severe acceleration of hypertension is required to produce such an extensive edema. In addition, the superimposed sudden ischemia induced by non-ST elevation myocardial infarction and uremia may aggravate the brain tissue edema. ${ }^{7,89}$ Ischemia and uremia damage the brain tissue, first by producing cytotoxic edema, and then extracellular edema because of ATP depletion. ${ }^{7,8,10}$

The difference in the level of arterial blood pressure for the formation of reversible edema between the cortical and subcortical area and the deep brain structures may be due to the different conditions of autoregulatory adjustment of cerebral blood flow. In animal studies, the upper limit of the autoregulatory plateau of cerebral blood flow in the thalamus has been shown to be higher than that in the cortex. ${ }^{11}$ The deep brain structures are fed by arterioles directly from the middle cerebral trunk or basilar artery and the cortical and subcortical area are fed by terminal branch arteries. Thus, the former regions are constantly subjected to high arterial pressure. Therefore, severe acceleration of hypertension may be necessary for the dysfunction of autoregulation and breakdown of the blood brain barrier to produce the more extensive vasogenic edema in such patients. ${ }^{12}$ The current case presented with acute renal failure precipitated by urinary tract infection and found to have severe hypertension. The MRI showed extensive edema throughout the whole brain with sparing of grey matter. These findings appeared to be causally related to each other.

In addition, there are several causes of diffuse cerebral hypodensities. This includes various neurological conditions, for example stroke, cerebral venous thrombosis, encephalitis and demyelinating disorder. Treatment with cytotoxic agents like cisplatin, calcineurin inhibitors, interferon and filgrastin may also precipitate diffuse cerebral edema. Other possible diagnose include AIDS and thrombotic microangiopathy. None of these causative factors was related to our patient. Other precipitating causes include severe metabolic abnormalities that disturbed the integrity of the brain vasculature or sympathetic activity such as ischemic bowel disease, sepsis, hyponatremia and renal dysfunction. In pregnant women, this abnormality can occur in the presence of eclampsia. The fact that she was not pregnant excluded eclampsia as the cause.

In our patient, we hypothesize that the extensive and diffuse edema seen on MRI began with vasogenic edema primarily due to the severe acceleration of hypertension and aggravated by myocardial infarction and uremia producing secondary cytotoxic edema. In conclusion, it is important for physicians to be aware of this syndrome, as its recognition may obviate unnecessary diagnostic procedures. More importantly, as shown in this patient, the condition is reversible simply by prompt lowering of elevated blood pressure and avoidance of offending cytotoxic or immunosuppressive agents. If treatment is delayed, permanent damage to the affected areas of the brain is likely. Indeed, it is fortunate that this patient did not suffer any long-term neurological sequelae despite such an extensive white matter edema.

\section{References}

1. Oppenheimer BS, Fishberg AM. Hypertensive encephalopathy. Arch Intern Med 1928;41: 264-78.

2. Ram CV. Hypertensive encephalopathy: recognition and management. Arch Intern Med 1978;138:1851-3.

3. Vaughan CJ, Delanty N. Hypertensive emergencies. Lancet 2000;356:411-7.

4. Schwartz RB, Jones KM, Kalina P et al. Hypertensive encephalopathy; finding on CT, MR imaging and SPECT imaging in 14 cases. AJR Am J Roentgenol 1992;159:37983.

5. Hinchey J, Chaves C, Appignani B et al. A reversible posterior leukoencephalopathy syndrome. N Engl J Med 1996;334:494500.

6. Hauser RA, Lacey DM, Knight MR. Hypertensive encephalopathy: magnetic resonance imaging demonstration of reversible cortical and white matter lesions. Arch Neurol 1988;45:1078-83. 
7. Dinsdale H. Hypertensive encephalopathy. Neurol Clin 1983;1:3-15.

8. Chester EM, Agamanolis DP, Banker BQ, Victor M. Hypertensive encephalopathy: a clinicopathologic study of 20 cases. Neurolgy 1978;28:928-39.

9. Perloff D. Hypertension and pregnancyrelated hypertension. Cardiol Clin
1998;16: 79-101.

10. Gokce M, Dogan E, Nacitarhan S, Demirpolat G. Posterior reversible encephalopathy syndrome caused by hypertensive encephalopathy and acute uremia. Neurocrit Care 2006;4:133-6.

11. Sadoshima S, Fujii K, Yao H et al. Regional cerebral blood flow autoregulation in normotensive and spontaneously hypertensive rats; the effects of sympathetic denervation. Stroke 1986;17:981-4.

12. Kumai Y, Toyoda K, Fujii K, Ibayashi S. Hypertensive encephalopathy extending into the whole brainstem and deep structures. Hypertens Res 2002;25:797-800. 